\title{
Time to fibrinolytics for acute myocardial infarction: Reasons for delays at Steve Biko Academic Hospital, Pretoria, South Africa
}

\author{
R Meel, MB ChB, MMed (Int Med), Cert Cardiol (CMSA); R Gonçalves, MB ChB, MMed (Int Med), Cert Cardiol (CMSA) \\ Department of Internal Medicine, School of Medicine, Faculty of Health Sciences, University of Pretoria, South Africa, and \\ Steve Biko Academic Hospital, Pretoria
}

Corresponding author: $R$ Meel (ruchikameel@gmail.com)

\begin{abstract}
Background. Fibrinolytic therapy is a time-critical intervention proven to reduce mortality and morbidity in patients with ST-elevation myocardial infarction (STEMI). Limited data exist in South Africa (SA) regarding time to fibrinolytic therapy for STEMI patients and reasons for delayed therapy.

Objectives. To establish the proportion of STEMI patients receiving fibrinolytic agents at Steve Biko Academic Hospital (SBAH), Pretoria, $\mathrm{SA}$, identify any delays to receiving fibrinolytic agents, and uncover reasons for those delays. The number of lives lost as a result of these delays was calculated.

Methods. This prospective, observational study included 100 consecutive patients presenting with a STEMI to SBAH. Using a researcheradministered questionnaire, the times from symptom onset to receipt of fibrinolytic therapy and the reasons for delays were documented. The number of lives lost was then calculated.

Results. Only $37 \%$ of patients received fibrinolytic therapy and only $3 \%$ received the medication within 1 hour. The median total delay in receiving fibrinolytic therapy was 270 minutes (range 45 - 584). The median time delays from onset of symptoms to call for help, between calling for help and arriving at hospital, and from hospital arrival to fibrinolytic agent administration, were 35 minutes (5 - 1185$), 55$ minutes (12.5 - 670) and 62.5 minutes (16.5 - 282), respectively. Numerous delays were identified at all stages, with patient and transport delays being most significant. Strikingly, an additional 32 patients per 1000 treated could have been saved if a fibrinolytic agent had been administered within 1 hour.

Conclusions. This study highlights the important problem of delayed or non-administration of fibrinolytic therapy at a tertiary hospital. The problems identified will contribute to the implementation of a robust STEMI management network in SA, similar to those in developed countries.
\end{abstract}

S Afr Med J 2016;106(1):92-96. DOI:10.7196/SAMJ.2016.v106i1.9801

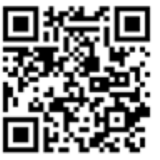

ST-elevation myocardial infarction (STEMI) is most commonly caused by the total occlusion of an epicardial coronary artery by a thrombus. ${ }^{[1]}$ The cornerstone of STEMI management is early revascularisation, either by primary percutaneous coronary intervention or fibrinolytic therapy, in conjunction with other adjunctive pharmaceutical agents. ${ }^{[1]}$ The most important determinant of outcome, irrespective of which form of revascularisation is selected, is the time from symptom onset to restoration of flow in the obstructed artery. ${ }^{[2]}$ The ideal reperfusion strategy for STEMI is primary percutaneous coronary intervention (PCI), provided it is performed in an experienced centre within 90 minutes of the time of hospital arrival or arrival of the emergency medical services (EMS) ${ }^{[1]}$ Fibrinolytic therapy should be administered as an alternative within 30 minutes, if primary PCI is unavailable or transport times exceed 60 minutes to the nearest centre capable of performing PCI. ${ }^{[1]}$ Randomised controlled trials of fibrinolytic therapy have demonstrated the benefit of initiating fibrinolytic therapy as early as possible after the onset of angina. ${ }^{[3,4]}$ A myocardial infarction may be aborted and mortality dramatically reduced if fibrinolytic therapy is administered within the first 2 hours, and particularly within the first hour. ${ }^{[2]}$

Delays relating to patient factors, emergency service and transport factors, and in-hospital factors broadly account for the delays in provision of appropriate reperfusion therapy. Globally, numerous studies have demonstrated that significant delays exist in the provision of any reperfusion strategy, including fibrinolytic therapy, to patients presenting with STEMI. ${ }^{[5-10]}$ The largest time delay is attributed to delays by the patient in seeking medical attention. Further delays occur following the call for help and relate to delays in transportation, as well as in-hospital delays.

In South Africa (SA) only a few tertiary public centres and selected private hospitals, almost exclusively in urban areas, are capable of performing primary PCI. The cardiology department at Steve Biko Academic Hospital (SBAH), Pretoria, was unable to perform routine primary PCI at the time of the study. Fibrinolytic therapy therefore constituted the primary revascularisation modality for patients presenting with STEMI. Furthermore, based on observation, few if any patients receive prehospital fibrinolytic therapy in SA, and there are minimal published data regarding the time to provision of fibrinolytic therapy (either prehospital or in-hospital). We therefore embarked on this study to systematically document the aforementioned observations in a tertiary hospital.

\section{Objectives and methods}

This prospective, observational study was performed over a 14-month period between August 2008 and November 2009 at SBAH. The sample comprised 100 consecutive patients presenting to SBAH with STEMI. All patients with STEMI according to the new universal definition of myocardial infarction were included ${ }^{[11]}$ unless 
fibrinolytic therapy was contraindicated or primary PCI was performed. This study only addressed fibrinolytic therapy and not adjunctive agents such as aspirin. At the time of the study, SBAH was the only public tertiary referral hospital with a cardiology service for northern Gauteng and Mpumalanga provinces. Additionally, uninsured patients presenting to private hospitals were also referred to SBAH for further management. Patients at the time could not receive primary PCI or rescue PCI services, making timeous fibrinolytic administration essential.

Data in respect of demographics, timing of fibrinolytic therapy and reasons for delays in therapy were captured with the aid of a questionnaire. The questionnaire was personally administered by the first author during the hospital admission to reduce recall bias. Relevant information was also retrieved from the patients' files, and via telephonic or personal interviews with the attending doctors where required. The total time delay between onset of symptoms and administration of fibrinolytic therapy was determined in minutes. Times were recorded to the nearest 5 minutes. A delay was defined as more than 60 minutes having elapsed from the time of symptom onset to the initiation of fibrinolytic therapy. ${ }^{[3]}$ Only the data for patients receiving the medication within 12 hours of onset of symptoms were analysed for in-hospital delays. However, these patients were included to analyse the prehospital delays.

The total time delay was then divided into: (i) patient factors - time from onset of symptoms to call for help: (ii) prehospital factors - time from call for help to arrival of help, and time from arrival of help to arrival at the hospital; and (iii) in-hospital factors door to assessment by a doctor, and time from assessment by a doctor to fibrinolytic therapy.

Potential reasons for delays were identified from the information obtained by the patient and patient record. Subsequently the impact of the delays in terms of loss of potential benefit (i.e. potential number of lives saved per thousand patients treated) was calculated from the above data.

\section{Statistical analysis}

All statistical analysis was performed using Matlab (version 7.1, MathWorks, USA). Categorical data were expressed as percentages. Continuous variables were expressed as means and standard deviations. Non-parametric data were expressed as medians and interquartile ranges.
The loss of absolute benefit for the sample, as a mean, was also calculated utilising the data from Boersma et al. ${ }^{[2]}$ The benefit of fibrinolytic therapy was $65,37,26$ and 29 lives saved per 1000 treated patients in the 0 - 1-, 1 - 2-, 2 - 3- and 3-6-hour intervals, respectively. ${ }^{[2]}$ Based on the nonlinear regression equation $f(x)=19.4$ $0.6 x+29.3 x^{-1}$, the loss of absolute benefit per 1000 patients treated, expressed as a percentage, is $L(x)=100(1-f(x) / f(1)) .{ }^{[2]}$ The loss of benefit relative to the first hour, as a percentage, can then be calculated as a mean for the 1-12-hour period. This value can be used to calculate the potential number of lives that could have been saved per 1000 patients treated for the 1 -12-hour group, compared with the first hour (recalling that 65 lives were saved per 1000 patients treated in the first hour). ${ }^{[2]}$

\section{Ethics}

Ethics approval was granted by the Faculty of Health Sciences Ethics Committee, University of Pretoria. The study was conducted in accordance with the Declaration of Helsinki.

\section{Results}

Baseline characteristics of the study patients are summarised in Table 1. The majority were white, male and had secondary level education. Seventy-three percent were employed. Fig. 1 outlines the referral course of the patients and the fibrinolytic therapy administration pattern. No patient received prehospital fibrinolytic therapy, and only $37 \%$ of eligible patients received fibrinolytic therapy at all. Thirty-nine patients appropriately did not receive the medication, because of delayed presentation (10 at the presenting hospital and 29 at SBAH). Only 3 patients received a fibrinolytic agent within 1 hour, 34 did so between 1 and 12 hours, and 63 either did not receive a fibrinolytic therapy at all or received it after 12 hours. Table 2 summarises the mean treatment delays in minutes.

\section{Prehospital delays}

In respect of prehospital delays, the mean time from onset of symptoms to call for help was 35 minutes (range 5 - 1 185). Sixty patients called for help between 0 and 1 hour, 33 between 1 and 12 hours, and 7 after 12 hours. The last group were not candidates for fibrinolytic therapy.

The most common reason for delay (37\%) was misinterpretation of symptoms as being non-cardiac in nature. Many patients (13\%) adopted a wait-and-see approach, hoping that the symptoms would disappear spontaneously. Many of these patients reported that they were not aware of the importance or availability of receiving rapid treatment. Some also tried self-medicating (4\%). Patients who had had a previous heart attack (3\%) were likely to accurately recognise their symptoms as being a possible myocardial infarction and seek help sooner.

Patient location did not influence helpseeking behaviour, except for patients who were driving. This latter group generally continued to their destination before seeking help.

Importantly, the vast majority (84\%) of patients did not call for an ambulance at all, instead opting to use a private vehicle to reach the hospital, which was immediately available in $86 \%$ of cases.

The reasons cited for not calling an ambulance included ease of access and use of patients' own transport, unfamiliarity with the emergency numbers, distrust of the emergency services (viewed as being slow,

Table 1. Baseline characteristics

\begin{tabular}{ll}
\hline Age (years), mean (range) & $52(30-84)$ \\
Gender male (\%) & 70 \\
Race (\%) & \\
Asian & 9 \\
Black & 14 \\
White & 74 \\
Coloured & 3 \\
Highest education level (\%) & \\
None & 2 \\
Primary & 8 \\
Secondary & 77 \\
Tertiary & 13 \\
Employment (\%) & \\
Employed & 73 \\
Unemployed & 9 \\
Pensioner & 18
\end{tabular}

Table 2. Summary of treatment delays (minutes), mean (range)

\begin{tabular}{ll}
\hline $\begin{array}{l}\text { Onset of symptoms } \\
\text { to call }\end{array}$ & $35(5-1185)$ \\
$\begin{array}{l}\text { Call to hospital } \\
\text { Call to arrival of help }\end{array}$ & $55(12.5-670)$ \\
$\quad \begin{array}{l}\text { Arrival of help to } \\
\text { hospital }\end{array}$ & $30(10-435)$ \\
$\begin{array}{l}\text { Door to fibrinolytic } \\
\text { Door to doctor }\end{array}$ & $62.5(16.5-282)$ \\
$\quad$ Doctor to fibrinolytic & $30(10-654)$ \\
Total delay & $270(45-584)$
\end{tabular}


inefficient and occasionally not responding at all), lack of understanding concerning the potential risks (e.g. ventricular fibrillation) associated with a myocardial infarction and the ability of paramedics to treat these problems, and lack of understanding of the availability of and need for early treatment.

When patients did call for an ambulance, its late arrival, either due to the EMS being overburdened or to the driver getting lost, further contributed to delays in many cases. None of the patients who called for an ambulance received prehospital fibrinolytic therapy.

Ninety-one percent of patients who called for help accessed transport within 1 hour. The majority of patients $(76 \%)$ arrived at the first hospital within 1 hour. The most common reasons for delay were long distances travelled between the scene and the hospital, presenting to a general practitioner or clinic first (18\%), and traffic delays. The majority (69\%) of patients were driven to hospital in a private vehicle, while $14 \%$ drove themselves. Only 16\% used an ambulance, and one patient walked to hospital.

\section{In-hospital delays}

A total of 38 patients were initially seen at primary or secondary level state hospitals, 20 at private hospitals and 42 at SBAH. The 58 patients who initially presented to other hospitals took a median of 8 hours (range 1 - 288) to be transferred to SBAH. The median time from onset of symptoms to first medical contact was 135 minutes (range 20 - 1400 ) or 2.3 hours (range 0.3 - 23.3). Only 34 patients arrived within 1 hour and 56 arrived between 1 and 12 hours after symptom onset. Ten of the patients were only seen 12 hours after the onset of symptoms and were therefore not candidates for fibrinolytic therapy; they were excluded from the in-hospital analysis of delays. Overlapping reasons for delays were noted for each of the components evaluated.

Of patients seen at referral hospitals, 32 (64\%) were seen within 30 minutes. Only 4 out of a potential 50 patients $(8 \%)$ received a fibrinolytic agent, all within 20 minutes of seeing a doctor. Three patients received fibrinolytic agents in the emergency room and one in an intensive care unit. Three

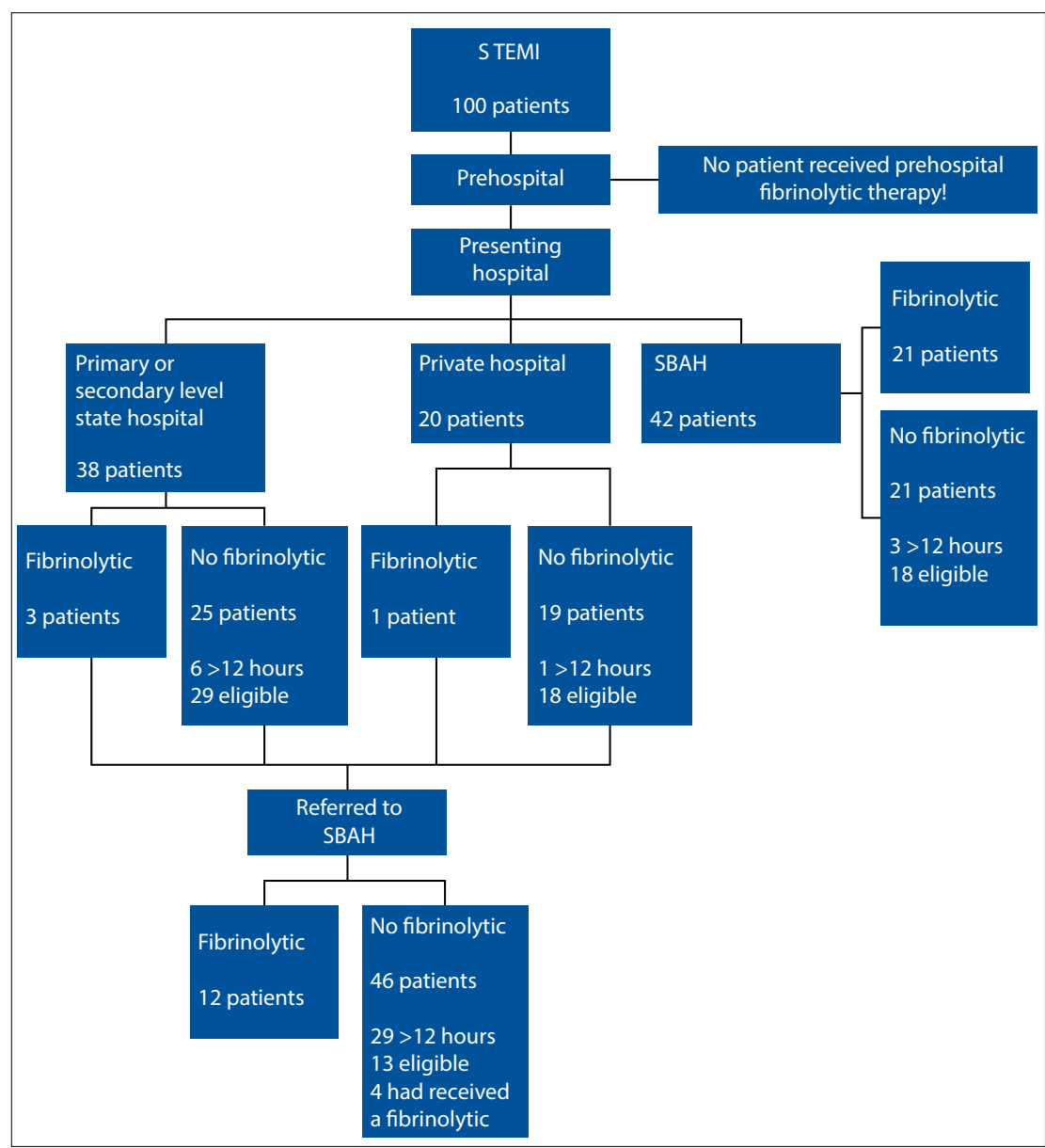

Fig. 1. Flow diagram of fibrinolytic administration patterns. Only $37 \%$ of patients received fibrinolytic therapy within 12 hours. patients received streptokinase and one alteplase. Total delay from symptom onset to treatment was 45 minutes for 3 patients and 430 minutes for one patient. Generally, decisions on fibrinolytic administration were made by casualty officers following telephonic consultation with an internal medicine registrar rotating through cardiology.

The most common reasons for delays in seeing a doctor were inappropriate triage with no priority given to patients with chest pain, waiting in queues to open folders prior to being evaluated, understaffing of hospitals with busy emergency rooms, and underresourced hospitals (electrocardiograph (ECG) machines were occasionally not working in some hospitals).

The most common reasons for delays in receiving fibrinolytic therapy were misdiagnosis of STEMI (including delays waiting for cardiac enzymes despite STEMI being obvious on the ECG or due to misinterpretation of the ECG), denial of fibrinolytic therapy on financial grounds in patients presenting to private hospitals, lack of familiarity with treatment protocols and inappropriate decision-making among attending doctors, especially inexperienced doctors, fear of complications, lack of urgency in administering the agents, absence of fibrinolytic agents in the emergency room or hospital, and late patient presentation (after 12 hours).

At SBAH 66 patients (83\%) were seen within 30 minutes. The reasons for delays in being assessed by a doctor were similar to those in the presenting hospitals. Only $58 \%$ of eligible patients received fibrinolytic agents within 12 hours. Twenty-seven patients received streptokinase while 5 received alteplase. Seven patients were given fibrinolytic therapy after 12 hours and were not included in the analysis.

At SBAH, after assessment by a doctor, $60 \%$ received a fibrinolytic agent within 1 hour of assessment, with $41 \%$ receiving the medication after more than 1 hour. Twentyfive patients received the fibrinolytic therapy in the emergency centre (EC), while 7 received it in the coronary care unit (CCU). The median time delay between hospital arrival and arrival in the CCU was 420 minutes (range 10 - 2 960). The door-tofibrinolytic time was a median of 60 minutes for those receiving the medication in the EC compared with 85 minutes for those receiving the medication in the CCU. The common reasons for delays in receiving fibrinolytic therapy were again similar to the other hospitals. Additionally, delayed referral from the presenting hospital contributed significantly. The decision to administer a 
fibrinolytic was generally made by either the casualty officer or an internal medicine registrar rotating through cardiology. (Owing to staff constraints, not all STEMI patients could be seen immediately by a registrar in internal medicine.)

Fig. 2 summarises the loss of absolute benefit as a percentage (i.e. the preventable 35-day mortality) for time delays beyond 1 hour but less than 12 hours. An analysis was performed to determine the loss of absolute benefit as a mean for the sample by utilising the data from Boersma et al. ${ }^{[2]}$ The mean loss of benefit as a percentage, relative to the first hour, for the $1-12$-hour period is $50.6 \%$. This value was used to determine the additional number of lives that could have potentially been saved per 1000 patients treated for the 1 - 12-hour group compared with the first hour. This value was 32/1 000 . An additional 32 patients per 1000 treated could therefore have been saved if the treatment had been administered within the first hour.

\section{Discussion}

The majority of patients in this study (67\%) did not receive fibrinolytic therapy at all. Furthermore, the majority of those who did receive treatment received it late. A large number of eligible patients arrived at a facility capable of providing fibrinolytic therapy, but did not receive any treatment. These missed opportunities are clearly resulting in excess mortality and morbidity, including heart failure, which would otherwise be preventable.

Total treatment delays and the components of prehospital and in-hospital treatment delays were all prolonged in comparison with the international literature. The largest cumulative delays were experienced as a result of predominantly prehospital factors. With regard to patient factors, delayed time ( $>1$ hour) from the onset of symptoms to call for help contributed significantly to delays in $40 \%$ of patients. The overriding reasons for these delays were lack of awareness of the symptoms of a myocardial infarction and how to respond appropriately and rapidly. This knowledge emphasises the importance of public awareness campaigns, as part of creating a STEMI network approach.

Of concern was that only 16 patients called for an ambulance, none of whom received prehospital fibrinolytic therapy. This represents an important opportunity for intervention by introducing paramedic training and certification to allow for prehospital fibrinolysis. Additionally, public awareness campaigns should focus on the risk of sudden death due to arrhythmias,

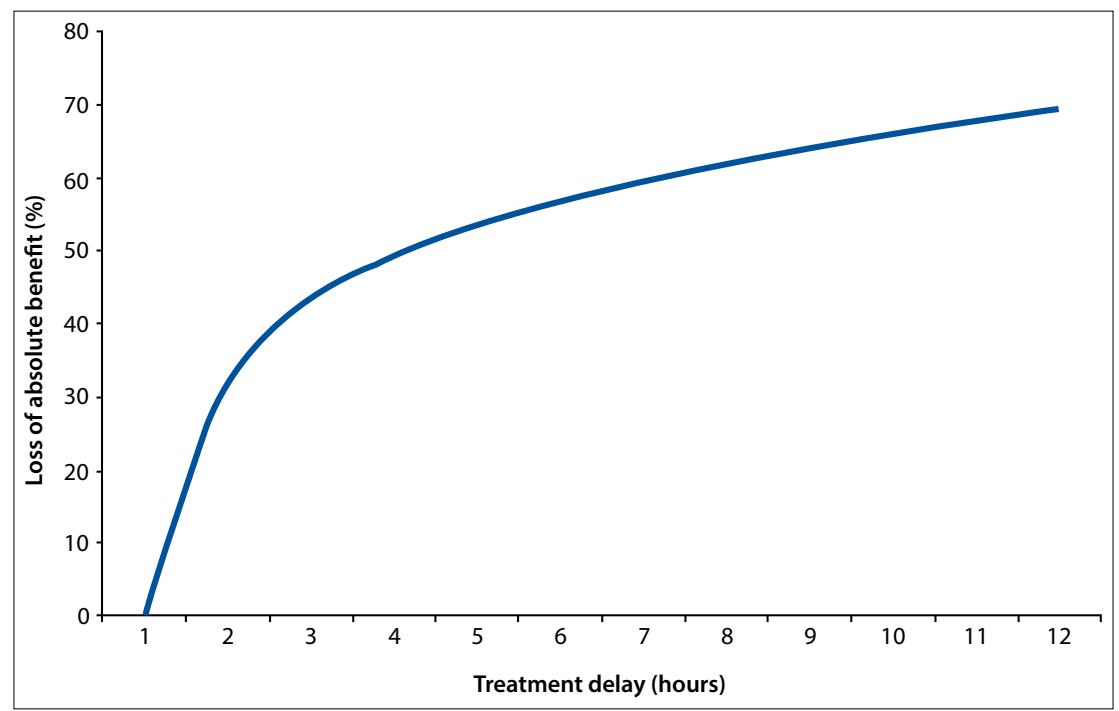

Fig. 2. Loss of absolute benefit (\%) v. treatment delay (hours).

which may be reduced by appropriately trained first responders. Patient perception of an inefficient EMS was also of concern. This may stem from the absence of a protocol-driven service, as well as poor resourcing, understaffing or poor training, although this requires further investigation. Integration of the EMS, including all first responders such as firemen and police officers, into the STEMI network would be crucial to its success. Staffing the receiving hospitals with full-time qualified staff to receive an ECG and then advise on therapy should become the standard of care. Having a clear plan regarding where to transport a patient within a given geographical area and the route of transportation is key. The geographical and financial constraints in SA dictate that early fibrinolytic therapy, ideally prehospital, would be the most efficient revascularisation strategy.

In-hospital delays, although notaccounting for the greatest proportion of the total delay, were important because of the fact that the median door-to-fibrinolytic time was more than double the current recommendation of 30 minutes. Large numbers of eligible patients were denied therapy despite arrival within 12 hours. Common themes emerged when reasons for these delays were explored. These reflect practices that are contradictory to the current guidelines. The dominant problems were related to poor systems of care and staffing and, less commonly, lack of resources. Denying STEMI patients presenting to private hospitals fibrinolytic therapy cannot be justified at all, as this constitutes part of the emergency care of these patients as mandated by law. This again emphasises the need for protocol-driven care in the management of STEMI patients.
The impact of having no system of care is clear. The ubiquitous lack of urgency on the part of staff, reflecting lack of knowledge, being over-worked or simply apathy, was disturbing. Inappropriate triage and registration delays were almost universal because of the lack of dedicated chest pain units. Furthermore, the first attending doctor who was appropriately able to administer a fibrinolytic agent generally did not do so. This should clearly be done at the hospital to which the patient presented first. Inappropriate diagnosis and lack of familiarity with fibrinolytic therapy and the management of the complications associated with its administration may explain this behaviour. Furthermore, the perception by many casualty officers that administering fibrinolytics is 'not their responsibility' should be addressed. In this regard, cardiology consultation was often sought in unambiguous cases, rather than initiating appropriate treatment. These deficiencies can clearly be improved by implementing a STEMI network of care that integrates chest pain units at receiving hospitals.

Other opportunities for improvement were identified. These include not ordering unneeded tests unless the diagnosis was clearly in question, and improving ECG interpretation skills in general. The introduction of bedside echocardiography could rapidly help to classify equivocal cases. However, this was seldom performed because of skills and equipment deficiencies. Interhospital transfer delays, while clearly problematic, could be mitigated if fibrinolytic agents were rapidly administered at the receiving hospital.

It is expected that public tertiary centres around SA may have a similar experience. 
Furthermore, based on personal observation, it is expected that many patients seen at the primary level of care are either missed or not appropriately referred at all. Owing to inappropriately constrained tertiary hospital capacity, patients are also frequently not accepted, although the exact numbers are difficult to estimate. Further research should be conducted in this regard.

\section{Study limitations}

This study represents the experience of a single tertiary centre. Referral bias is clearly a concern. Additionally, patients presenting after 12 hours were included in the study although they had presented late for fibrinolytic therapy. This was done in order to demonstrate the 'real-world experience' in SA and account for the reasons behind these patient delays. Other limitations were recall bias on the part of the patient and occasional lack of documentation of the times of in-hospital events in the notes. The EMS scene time, door-toECG time and ECG-to-decision-to-give-fibrinolytic time were not routinely recorded in the available patient records.

\section{Conclusion}

Many patients failed to benefit from fibrinolytic therapy owing to a complete lack of fibrinolytic administration, delayed presentation and delayed therapy. This study highlights both the challenges and the opportunities that must be grasped in order to improve patient outcomes. Recognition of the seriousness and extent of this disease in the midst of numerous other healthcare priorities in SA is crucial. The authors encourage an urgent commitment to address these issues by all involved stakeholders. The implementation of a STEMI network, modified to local practice, is as possible as it is urgent.
Acknowledgements. We thank Dr Rita Sommers, Dr Shiraz Ellimdin, Dr Chris Mostert and Prof. James Ker (senior) for their valuable advice and for reviewing the manuscript. Furthermore, we appreciate the help provided by the staff of the coronary care unit at SBAH. Thanks also to Dr Duarte Gonçalves, $\mathrm{PhD}$, for his assistance with the statistics.

\section{References}

1. Antman EM, Anbe DT, Armstrong PW, et al. ACC/AHA guidelines for the management of patients with ST-elevation myocardial infarction. Circulation 2004;110(15):588-636. [http://dx.doi. org/10.1161/01.CIR.0000134791.68010.FA]

2. Boersma E, Mercado N, Poldermans D, et al. Acute myocardial infarction. Lancet 2003;361(9360):847858. [http://dx.doi.org/10.1016/S0140-6736(03)12712-2]

3. Gruppo Italiano per Io Studio della Streptochinasi nell Infarto miocardico (GISSI). Effectiveness of intravenous thrombolytic treatment in acute myocardial infarction. Lancet 1986;327(8478):397-402. [http://dx.doi.org/10.1016/S0140-6736(86)92368-8]

4. Fibrinolytic Therapy Trialists (FTT) Collaborative Group. Indications for fibrinolytic therapy in Fibrinolytic Therapy Trialists (FTT) Collaborative Group. Indications for fibrinolytic therapy in
suspected acute myocardial infarction: Collaborative overview of early mortality and major morbidity suspected acute myocardial infarction: Collaborative overview of early mortality and major morbidity
results from all randomized trials of more than 1000 patients. Lancet 1994;343(8893):311-322. [http:// dx.doi.org/10.1016/S0140-6736(94)91161-4]

5. Birkhead J. Trends in the provision of thrombolytic treatment between 1993 and 1997. Myocardial Infarction Audit Group. Heart 1999;82(4):438-442. [http://dx.doi.org/10.1136/hrt.82.4.438]

6. Doyle F, de la Harpe D, McGee H, et al. Nine-year comparison of presentation and management of acute coronary syndromes in Ireland: A national cross-sectional survey. BMC Cardiovasc Disord 2005;5(5):1-8. [http://dx.doi.org/10.1186/1471-2261-5-5]

7. Davies C, Christenson J, Campbell A, et al. Fibrinolytic therapy in acute myocardial infarction: Time to treatment in Canada. Can J Cardiol 2004;20(8):801-805.

8. Hirvonen T, Halinen M, Kala R, Olkinuora J. Delays in thrombolytic therapy for acute myocardial infarction in Finland: Results of a national thrombolytic therapy delay study. Finnish Hospitals ${ }^{3}$ Thrombolysis Survey Group. Eur Heart J 1998;19(6):885-892. [http://dx.doi.org/10.1053/euhj.1997.0866 885-892]

9. Gibson C, Pride Y, Frederick P, et al. Trends in reperfusion strategies, door-to-needle and door-toballoon times, and in-hospital mortality among patients with ST-segment elevation myocardial infarction enrolled in the National Registry of Myocardial from 1990 to 2006. Am Heart J infarction enrolled in the National Registry of Myocardial from

10. Eagle K, Nallamothu B, Mehta R, et al. Trends in acute reperfusion therapy for ST-segment elevation myocardial infarction from 1999 to 2006: We are getting better but we have got a long way to go. Eur
motion Heart J 2008;29(5):609-617. [http://dx.doi.org/10.1093/eurheartj/ehn069 609-617]

11. Thygesen K, Alpert J, White H, et al. Universal definition of myocardial infarction. Circulation 2007;116(22):2634-2653. [http://dx.doi.org/10.1161/CIRCULATIONAHA.107.187397] 